\section{Desempenho da Potência Anaeróbia em Atletas de Elite do Mountain Bike Submetidos à Suplementação Aguda com Creatina}

\author{
Anaerobic Power Output of Elite Off-road Cyclists With Acute Oral \\ Creatine Supplementation
}

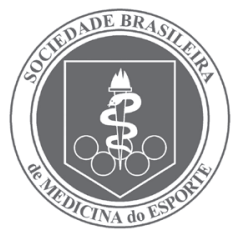

Artigo Original

\begin{abstract}
Guilherme Eckhardt Molina Guilherme Fávero Rocco ${ }^{2}$ Keila Elizabeth Fontana ${ }^{1,2}$
\end{abstract}

1. Programa de Pós-Graduação em Ciências da Saúde - Universidade de Brasília.

2. Programa de Pós-Graduação em Educação Física - Universidade de Brasília.

\section{Endereço para correspondência:}

Guilherme Eckhardt Molina

QRSW quadra 01, Bloco B-12 apt.

202 Sudoeste

70675-132 - Brasília, DF, Brasil.

E-mail: gmolina@unb.br

Submetido em: 09/10/2008

Versão final recebida em: 12/05/2009

Aceito em: 13/05/2009

\begin{abstract}
RESUMO
Com o objetivo de investigar os efeitos da suplementação aguda com creatina no desempenho da potência anaeróbia de atletas de elite do mountain bike, 20 atletas em período básico do macrociclo de treinamento foram distribuídos aleatoriamente (duplo-cego) em dois grupos: placebo (PLA, n=10) e creatina (CRE, $n=10$ ). Foram avaliados quanto à composição corporal (pesagem hidrostática) e potência anaeróbia (teste de Wingate - TW) antes (PRÉ) e depois (PÓS) de sete dias de suplementação. A creatina ou maltodextrina foi usada em três doses diárias de 0,3g/kg de massa corporal diluídos em meio líquido adoçado. Não foram observadas diferenças significativas nas variáveis morfológicas após sete dias de suplementação (PRÉ x PÓS), e os grupos não diferiram apesar da variação percentual $(\triangle \%)$ contrária (positiva para o grupo CRE e negativa para o PLA). A potência anaeróbia pico (PP) e o instante da potência pico (IPP) aumentaram e o índice de fadiga diminuiu do PRÉ para o PÓS-testes no grupo CRE, enquanto que o grupo PLA não apresentou diferenças significantes. A PP apresentou forte tendência em ser maior e o IPP foi maior no grupo CRE comparado com o PLA. Conclui-se que existem evidências de que a suplementação com creatina $(0,3 \mathrm{~g} / \mathrm{kg})$ em curto prazo (sete dias) pode retardar o IPP (CRE 3,0 \pm 0,5/3,6 \pm 0,8 $\Delta \%=20 \%$ ) no teste de Wingate em atletas de elite do mountain bike, sugerindo que a suplementação com creatina pode melhorar o desempenho físico quanto à potência anaeróbia durante o trabalho de alta intensidade e curta duração.
\end{abstract}

Palavras-chave: teste de Wingate, pesagem hidrostática, composição corporal.

\begin{abstract}
In order to investigate the effect of a high dose, acute oral creatine supplementation on anaerobic power of male off-road cyclists, twenty elite athletes training at the basic period were randomly (double-blind) assigned into 2 groups: placebo (PLA $n=10$ ) and creatine (CRE $n=10)$. They were submitted to a body composition evaluation (underwater weighting) and Wingate Anaerobic Test (TW) before (PRE) and after (POS) seven days of supplementation. The oral supplements (creatine and maltodextrine) were taken in sweetened vehicle with three equal doses per day $(0.3 \mathrm{~g} / \mathrm{kg}$ body mass/dose). After a seven-day period, no significant differences were found on morphological variables (PRE x POS) and there was no difference between the groups in spite of the opposite percent variation ( $\triangle \%)$ (positive for CRE and negative for PLA group). The anaerobic power output (PP) and the moment of pick power (IPP) increased and the fatigue index decreased from PRE to POST tests in CRE group; however, the PLA group did not show significant differences. The PP showed strong tendency to increased values and IPP was greater in the CRE compared with the PLA group. The results of the present study indicated that short-term creatine supplementation $(0.3 \mathrm{~g} / \mathrm{kg})$ can postpone the anaerobic IPP (CRE 3.0 \pm 0.5/3.6 $\pm 0.8 \Delta \%=20 \%$ ) leading to significant improvement on the performance of power output and peak power moment in off-road cyclists.
\end{abstract}

Keywords: Wingate anaerobic test (WANT), underwater weighting, body composition.

\section{INTRODUÇÃO}

A creatina vem sendo usada ao longo de mais de duas décadas por indivíduos fisicamente ativos e atletas de diversas modalidades, devido aos seus possíveis efeitos ergogênicos sobre o desempenho anaeróbio e na hipertrofia muscular ${ }^{(1,2)}$.

Existem fortes evidências de que a quantidade de creatina fosfato (CP) estocada nos músculos possa ser o fator limitante do desempenho físico em exercícios de alta intensidade e curta duração ${ }^{(3,4)}$. Dessa forma, o aumento nos estoque de CP e creatina total (CT) através da suplementação com creatina pode aumentar a oferta de CP e consequen- temente aumentar a ressíntese de adenosina trifosfato (ATP) em até 30\%, além de auxiliar na capacidade de tamponamento dos músculos, favorecendo a melhoria do desempenho nesse tipo de exercício(5).

Uma modalidade esportiva pouco estudada e que pode beneficiar-se do potencial ergogênico da creatina é o mountain bike cross country, modalidade olímpica que demanda alta intensidade durante as competições, pois as provas são em circuito de terra, misturando subidas, descidas e retas, exigindo do atleta inúmeras explosões musculares ao longo do percurso como estratégia de ataque e ou defesa, além da necessidade de recuperação após o trabalho físico, gerado em diversas intensidades de esforço ${ }^{(6)}$. 
Atualmente para se quantificar essas respostas fisiológicas em laboratório, adota-se o teste de Wingate (TW), que apresenta alta reprodutibilidade, validade e fácil aplicação. O TW é um método não invasivo que também é amplamente utilizado para investigar o potencial efeito ergogênico da creatina(7).

Diante dessas evidências acredita-se que o impacto da suplementação com creatina em esforço físico sob alta intensidade e curta duração, com o controle prévio do treinamento físico e manutenção dos hábitos alimentares, pode demonstrar o potencial efeito ergogênico dessa substância em atletas de elite do mountain bike. Portanto, o objetivo do presente estudo foi investigar os efeitos morfofuncionais da suplementação aguda com creatina em atletas de elite do mountain bike.

\section{MÉTODOS}

Foram selecionados voluntariamente para participar deste estudo 20 atletas profissionais de ciclismo da modalidade mountain bike com idade entre 18 e 34 anos, do sexo masculino. Todos os atletas tinham no mínimo três anos de treinamento na modalidade, estavam na fase básica do macrociclo de treinamento, responderam à anamnese constituída por histórico clínico pessoal e familiar, e não faziam uso de creatina e esteroides anabolizantes nos últimos seis meses. Todos assinaram o termo de consentimento livre e esclarecido, aprovado pelo Comitê de Ética em pesquisa da Faculdade de Ciências da Saúde da Universidade de Brasília, de acordo com a Resolução 196/96 do Conselho Nacional de Saúde (registro do projeto: 035/2005).

Os atletas foram submetidos inicialmente à avaliação da composição corporal e da potência anaeróbia e divididos aleatoriamente em dois grupos. O grupo experimental (CRE; $n=10)$ foi submetido à suplementação com creatina monoidratada (Creatine Powder, Optimum Nutrition - Illinois, EUA), enquanto o grupo controle (PLA; $n=10)$ recebeu placebo (maltodextrina - Integralmédica, Embu-Guaçu, SP). Durante os sete dias de experimento os atletas consumiram 0,3g/kg de massa corporal total de creatina ou placebo em três doses iguais ao longo do dia dissolvidas em $250 \mathrm{~mL}$ de bebida carboidratada ${ }^{(8)}$. Os suplementos foram acondicionados em embalagem plástica com cor e textura semelhantes, não permitindo a identificação do suplemento utilizado por cada atleta. A pesagem e distribuição dos suplementos ficaram sob a responsabilidade de um técnico do laboratório, garantindo o caráter duplo-cego do estudo.

Após os sete dias de suplementação os atletas retornaram ao laboratório para refazer as avaliações (reteste). Todos os atletas foram orientados a manter seus hábitos alimentares e rotina de treinos durante o período de suplementação, evitando qualquer outra e bebidas cafeinadas (café, chocolate, mate, pó de guaraná, bebidas energéticas, coca-cola e guaraná), uma vez que a cafeína parece anular o efeito da creatina ${ }^{(9)}$. A ingestão de água foi ad libitum.

A massa corporal total (MCT) foi medida em balança de plataforma digital com precisão de 50g (2096 PP, Toledo - São Paulo, SP) e a estatura em estadiômetro de madeira com precisão de $0,1 \mathrm{~cm}$. A pesagem hidrostática foi realizada em um tanque com a temperatura da água entre 32 e $35^{\circ} \mathrm{C}$, utilizando uma cadeira de tubo plástico presa a um dinamômetro (Filizola - São Paulo, SP) com precisão de 100g. Os atletas compareceram ao laboratório entre 7 e 9 horas da manhã, em jejum de seis horas e com bexiga e intestino vazios. Foi evitado o consumo de alimentos que produzissem gases nas 12 horas que antecederam a pesagem. Foram realizadas 10 medidas de pesagem subaquática com imersão completa e expiração máxima forçada para esvaziamento dos pulmões. Foi calculada a média aritmética das três maiores pesagens. A densidade corporal foi obtida de acordo com a fórmula proposta por Brozek et al. ${ }^{(10)}$ considerando a temperatura da água ${ }^{(11)}$ e os volumes: residual (VR) e gastrintestinal (VGI). OVR foi determinado pela equação de Goldman \& Becklake ${ }^{(12)}$, e o VGl estimado em 100ml, conforme proposto por Buskirk ${ }^{(13)}$. A densidade corporal foi então utilizada para determinar a percentagem de gordura corporal (GORD\%)(14).
O teste de Wingate (TW), por apresentar alta reprodutibilidade, validade, além de ser não invasivo e de fácil aplicação, é amplamente utilizado para investigar o potencial efeito ergogênico da creatina ${ }^{(15)}$ Assim, foi utilizado para medir a potência anaeróbia em um cicloergômetro de frenagem mecânica com sensor de leitura e de torque por segundo na roda (Biotec 1800, Cefise - Campinas, SP). O protocolo consistiu em um aquecimento de cinco minutos com a carga de $88 \mathrm{~W}$ e 90rpm. A cada minuto foi realizado um sprint de seis segundos como processo de familiarização ao teste. Após dois minutos de descanso, os atletas realizaram o teste pedalando durante 30 s na maior velocidade possível contra resistência fixa de 7,5\% da $\mathrm{MCT}^{(16)}$. Foram registradas a potência pico e potência média, ambas em valores relativos $\left(\mathrm{W}^{\mathrm{k}} \mathrm{kg}^{-1}\right)$, instante da potência pico (s) além do índice de fadiga (\%).

Para a caracterização da amostra foi utilizada a estatística descritiva com base nos cálculos de média aritmética e desvio padrão. ANOVA one way foi utilizada para verificar a distribuição aleatória dos grupos. O teste t de Student para amostras pareadas (dependentes) nos informou sobre as diferenças observadas nas condições PRÉ e PÓS suplementação ao nível de 5\% (análise intragrupo). Para análise das diferenças entre os procedimentos de suplementação (entre grupos) foi utilizada a análise de covariância (ANCOVA). Utiliza-se no processamento estatístico dos dados o programa Statistical Package for Social Sciences (SPSS 13.0).

\section{RESULTADOS}

Nenhuma diferença significativa $(p<0,05)$ foi encontrada entre os grupos CRE e PLA no início do experimento, o que demonstra homogeneidade entre os grupos (tabela 1).

Tabela 1. Características físicas e tempo de treinamento dos grupos suplementados (CRE e PLA)

\begin{tabular}{c|c|c|c|c|c|c}
\hline GRUPOS & $\mathrm{n}$ & $\begin{array}{c}\text { IDADE } \\
(\mathrm{anos})\end{array}$ & $\begin{array}{c}\text { MCT } \\
(\mathrm{kg})\end{array}$ & $\begin{array}{c}\text { ESTATURA } \\
(\mathrm{cm})\end{array}$ & $\begin{array}{c}\text { IMC } \\
\left(\mathrm{kg} \cdot \mathrm{m}^{-2}\right)\end{array}$ & $\begin{array}{c}\text { TREINO } \\
(\mathrm{anos})\end{array}$ \\
\hline PLA & 10 & $26,4 \pm 5,5$ & $69,7 \pm 7,6$ & $174,3 \pm 6,4$ & $22,8 \pm 1,1$ & $4,6 \pm 2,3$ \\
\hline CRE & 10 & $29,5 \pm 4,6$ & $68,3 \pm 8,7$ & $179,1 \pm 5,7$ & $21,2 \pm 1,9$ & $6,4 \pm 3,4$ \\
\hline
\end{tabular}

Valores: média \pm desvio padrão, MCT = massa corporal total, IMC= índice de massa corporal, PLA = grupo placebo, $\mathrm{CRE}=$ grupo creatina

As médias e seus respectivos desvios-padrões, bem como o nível de significância (intragrupo) e variação percentual estão descritos na tabela 2. Não foi observada nenhuma diferença significativa nas variáveis morfológicas após sete dias de suplementação para os grupos CRE e PLA. Observa-se ainda redução percentual de todas as variáveis morfológicas no grupo PLA, fenômeno esse observado somente nas variáveis GORD (\%) e GORD (kg) no grupo CRE. A MCT e MCM após suplementação com CRE demonstraram variação percentual positiva, ao passo que no grupo PLA observou-se variação negativa.

Tabela 2. Composição corporal antes e após sete dias com e sem suplementação.

\begin{tabular}{c|c|c|c|c|c}
\hline \multirow{2}{*}{ VARIÁVEIS } & GRUPO & PRÉ & PÓS & $P$ & $\Delta \%$ \\
\hline \multirow{2}{*}{ MCT $(\mathrm{kg})$} & PLA & $69,7 \pm 7,7$ & $69,2 \pm 7,2$ & 0,16 & $-0,7$ \\
\cline { 2 - 6 } & CRE & $68,4 \pm 8,7$ & $68,8 \pm 9,2$ & 0,23 & 0,5 \\
\hline \multirow{2}{*}{ GORD\% } & PLA & $12,2 \pm 0,8$ & $11,7 \pm 0,8$ & 0,54 & $-4,0$ \\
\cline { 2 - 6 } & CRE & $10,7 \pm 1,1$ & $9,7 \pm 0,9$ & 0,11 & $-9,2$ \\
\hline \multirow{2}{*}{ MCM $(\mathrm{kg})$} & PLA & $61,8 \pm 0,4$ & $61,1 \pm 2,1$ & 0,75 & $-1,1$ \\
\cline { 2 - 6 } & CRE & $61,1 \pm 2,2$ & $61,8 \pm 2,2$ & 0,18 & 1,1 \\
\hline \multirow{2}{*}{ GORD $(\mathrm{kg})$} & PLA & $8,5 \pm 2,0$ & $7,6 \pm 1,5$ & 0,23 & $-10,1$ \\
\cline { 2 - 6 } & CRE & $7,5 \pm 3,0$ & $6,3 \pm 1,8$ & 0,11 & $-15,4$ \\
\hline
\end{tabular}

Valores: média \pm desvio padrão, PRÉ = antes do experimento, $P O ́ S=$ depois do experimento, $P=$ probabilidade $(t-p a-$ reado), $\Delta \%=$ diferença percentual entre os resultados pré e pós, $M C T=$ massa corporal total, GORD\% $=$ percentual de gordura, $\mathrm{MCM}=$ massa corporal magra, $\mathrm{GORD}=$ massa de gordura, $\mathrm{PLA}=$ grupo placebo, $\mathrm{CRE}$ = grupo creatina 
O instante da potência pico (IPP) aumentou significativamente, ao passo que o índice de fadiga diminuiu após suplementação com creatina (tabela 3); também foi verificado aumento altamente significante $(p<0,01)$ da potência pico (intragrupo) após suplementação. Ainda na tabela 3, observa-se manutenção da potência anaeróbia pico e resistência, enquanto que o índice de fadiga e o IPP apresentaram redução percentual no grupo PLA após o período experimental.

Tabela 3. Índices fisiológicos obtidos antes e após sete dias com e sem suplementação

\begin{tabular}{c|c|c|c|c|c}
\hline VARIÁVEIS & GRUPO & PRÉ & PÓS & $P$ & $\Delta \%$ \\
\hline $\begin{array}{c}\text { Potência pico } \\
(\text { W·kg) }\end{array}$ & PLA & $11,3 \pm 0,7$ & $11,3 \pm 0,5$ & 0,95 & $-1,2$ \\
\cline { 2 - 6 } & CRE & $11,3 \pm 0,5$ & $11,7 \pm 0,5$ & ${ }^{* *} 0,01$ & 3,8 \\
\hline $\begin{array}{c}\text { Resistência } \\
\text { anaeróbia (W·kg) }\end{array}$ & PLA & $9,2 \pm 0,7$ & $9,2 \pm 0,5$ & 0,89 & $-0,2$ \\
\cline { 2 - 6 } & CRE & $9,2 \pm 0,2$ & $9,3 \pm 0,3$ & 0,19 & 1,6 \\
\hline $\begin{array}{c}\text { Indice de fadiga } \\
(\%)\end{array}$ & PLA & $36,7 \pm 5,4$ & $36,6 \pm 6,1$ & 0,96 & $-0,3$ \\
\cline { 2 - 6 } & CRE & $36,1 \pm 6,5$ & $33,3 \pm 7,5$ & ${ }^{*} 0,04$ & $-7,6$ \\
\hline \multirow{2}{*}{$\begin{array}{c}\text { IPP } \\
\text { (s) }\end{array}$} & PLA & $3,1 \pm 0,3$ & $2,9 \pm 0,2$ & 0,34 & $-2,5$ \\
\cline { 2 - 6 } & CRE & $3,0 \pm 0,5$ & $3,6 \pm 0,8$ & ${ }^{*} 0,02$ & 20,0 \\
\hline
\end{tabular}

Valores: média \pm desvio padrăo, PRÉ $=$ antes do experimento, PÓS $=$ depois do experimento, $P=$ probabilidade ( $t$-pareado), ${ }^{*}=$ significante para $P<0,05,{ }^{* *}=$ significante para $P<0,01, \Delta \%=$ diferença percentual entre os resultados pré e pós, $\mathrm{PLA}=$ grupo placebo, $C R E=$ grupo creatina, $I \mathrm{PP}=$ instante da potência pico.

Quando comparada a potência pico nos grupos CRE e PLA (ANCOVA), observou-se forte tendência $(p=0,053)$ no sentido de maior potência pico $\left(\mathrm{W} \cdot \mathrm{kg}^{-1}\right)$ no grupo suplementado com creatina (gráfico 1). Já o instante da potência pico foi maior para o grupo suplementado com creatina (gráfico 2). Nas demais variáveis da potência anaeróbia não foram observadas diferenças significativas entre os grupos após suplementação.

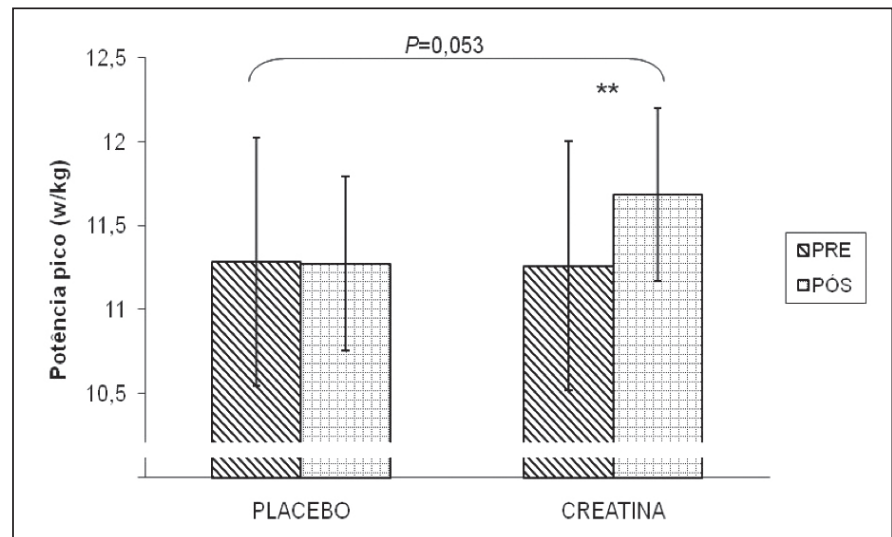

Gráfico 1. Potência pico antes (PRÉ) e depois (PÓS) de sete dias de treinamento com e sem suplementação de creatina.

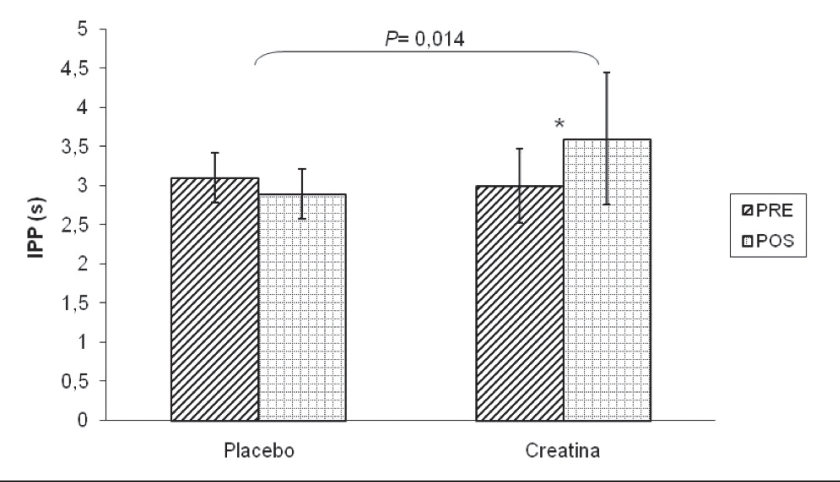

Gráfico 2. Instante da potência pico antes (PRÉ) e depois (PÓS) de sete dias de treinamento com e sem suplementação de creatina. As barras representam os valores médios e desvio-padrão.

\section{DISCUSSÃO}

Após o procedimento experimental não foram verificadas diferenças significativas intra e entre os grupos CRE e PLA para as variáveis morfológicas. Contudo, o grupo CRE demonstrou variação percentual positiva para as variáveis MCT e MCM, fenômeno esse não observado para o grupo PLA, que apresentou variação percentual negativa para as mesmas variáveis. Alguns estudos ${ }^{(17,18)}$ mostraram aumentos da MCT e MCM resultantes da suplementação aguda de CM em diversos grupos de indivíduos (sedentários, ativos, treinados e atletas) quando associados ao treinamento de força e/ou potência; entretanto, no presente estudo a MCT e MCM não diferiram significativamente. Chamamos a atenção para o fato de que a amostra foi composta por atletas de elite do mountain bike em fase de treinamento que não visava o ganho de força ou de potência; esse fato possivelmente justifica os resultados obtidos.

A restrição da suplementação à base de carboidratos e proteínas durante o procedimento experimental e o volume de treinamento (702 $\pm 39 \mathrm{~km} /$ semana) podem ter contribuído, potencializando um comportamento catabólico do grupo PLA descrito na tabela 2. Sabe-se que durante as sessões de treinamento, faz-se necessário o aumento de sinalizadores (hormônios) hiperglicemiantes na circulação para a manutenção do trabalho físico e controle glicêmico, podendo assim ativar o mecanismo de proteólise ATP-dependente (ubiquitina-proteossoma), o que pode explicar a redução da MCT e MCM no grupo PLA. Entretanto, no grupo CRE foi observada redução das variáveis GORD (\%) e GORD (kg).

Os exercícios de longa duração potencializam a capacidade de oxidar ácidos graxos de cadeia média e os aminoácidos podem participar como precursores de glicose e como intermediários do ciclo do ácido cítrico ${ }^{(19)}$, corroborando os índices morfológicos observados no grupo PLA após suplementação. Sabe-se que o mecanismo proteolítico ATP-dependente (ubiquitina-proteossoma) é inibido por fatores de crescimento como insulin growth factor $(\mathrm{GGF})^{(20)}$, o que pode justificar a diferença nas variações percentuais, uma vez que o aumento da transcrição gênica do RNAm para o IGF1 e IGF2 após cinco dias de suplementação com CM foi registrado e a suplementação com CM pode então mediar a sinalização para o anabolismo muscular ${ }^{(17)}$. Sabe-se ainda que a suplementação com CM em curto prazo aumenta o número de células satélites e mionúcleos ${ }^{(18)}$ e que a suplementação com CM até sete dias altera o conteúdo de água corporal e/ou reduz o volume urinário, através da estimulação por insulina e por transporte sódio- dependente. Cerca de $60-70 \%$ da CM são depositados como soluto creatina fosfato, no qual gera gradiente de sódio e aminoácidos através da membrana, que por sua vez potencializa a pressão osmótica, deixando o meio hipotônico.

Diferente do grupo CRE, o grupo PLA obteve redução dos índices morfológicos de MCT, MCM, GORD (\%) e GORD (kg), enquanto que o grupo CRE teve redução apenas na GORD (\%) e GORD (kg)(21).

As variações percentuais observadas nas variáveis morfológicas de ambos os grupos podem indicar que durante o período experimental a CM funcionou como um agente anticatabólico para o grupo CRE. Esse comportamento também foi observado nos índices funcionais avaliados no TW após suplementação.

A potência pico, ou seja, a maior potência registrada após suplementação, apresentou variação percentual positiva do PRÉ para o PÓS-teste no grupo CRE. A variação de 3,8\% foi significante $(p=0,01)$ quando comparada de forma pareada (intragrupo), corroborando os estudos de quatro dias de suplementação com CM (20g ao dia) de Tarnopolsky e Maclennan ${ }^{(22)}$, que verificaram aumento significativo $(p<0,05)$ de 3,7\% na potência pico $\left(W \cdot \mathrm{kg}^{-1}\right)$. Reen et al. ${ }^{(23)}$ verificaram 
que cinco dias de suplementação com CM (20g/dia) foram suficientes para gerar alterações significativas $(p<0,05)$ na potência pico durante sprints repetitivos em cicloergômetro. Quando comparada a potência pico nos grupos CRE e PLA (ANCOVA), observou-se forte tendência $(p=0,053)$ para maior potência pico $\left(W \cdot k^{-1}\right)^{-1}$ no grupo $C R E$ comparado com o grupo PLA (gráfico 1).

Sabe-se ainda, que a variância da amostra é sensível ao tamanho da mesma; sendo assim, o presente estudo ficou limitado a uma amostra pequena por se tratar de atletas de elite do mountain bike. Entretanto, o grupo CRE apresentou diferença significativa no instante de potência pico (IPP) ( $p=0,02)$ quando comparados intragrupo (PRÉ x PÓS), enquanto que o grupo PLA, não (tabela 3). O instante da potência pico foi significativamente $(p=0,02)$ retardado no grupo suplementado com creatina, o que vai ao encontro dos achados de Eckerson et al.(24). Quando comparados os grupos (CREX PLA), verificou-se diferença significativa $(p=0,014$ ) (gráfico 2$)$. Resultados semelhantes foram também observados por outros autores ${ }^{(25,26)}$ corroborando os da presente pesquisa quanto à potência pico e IPP.

A relação dos estoques de CP no músculo esquelético com a potência pico e IPP associam-se com a capacidade de manutenção das altas taxas de ressíntese de ATP pela hidrolise da CP durante o exercício intenso. Esses dados têm significativa $(p<0,05)$ correlação $(r=$ 0,70 ) entre a melhora do pico de potência muscular e a excreção de creatina urinária(27). Kurosawa et al..25), utilizando-se da espectroscopia, verificaram que após suplementação em curto prazo com CM os indivíduos ativos fisicamente apresentaram aumento de 65,0 $\pm 4,2 \%$ de degradação de CP e da taxa de síntese de ATP através da hidrólise de $\mathrm{CP}$ em tiro de velocidade, isso ocasionado pelo aumento significativo da concentração muscular de CP e do pH muscular.
De acordo com Chetlin et al. ${ }^{(28)}$ e Hoffman et al. ${ }^{(29)}$, a taxa de mobilização de CP no músculo é afetada pela intensidade e duração do exercício, concentração de CP no músculo e o tipo de fibra muscular envolvida no exercício. Em seus estudos com suplementação em curto prazo de CRE, demonstraram aumento significativo da concentração de CP nas fibras do tipo II. Elas são especialmente importantes nos exercícios de alta intensidade e baixa duração, devido ao fato de ser recrutadas com maior velocidade e em quantidade, além de morfologicamente desenvolvidas para a utilização do ATP e ressíntese de ATP durante o exercício intenso quando comparadas com as fibras do tipo I.

O índice de fadiga diminuiu 7,6\% ( $p<0,05)$ após suplementação. Essa redução do índice de fadiga é atribuída à quantidade extra de CP que tamponaria o fosfato inorgânico produzido na hidrólise do ATP, mantendo a concentração hidrogeniônica mais baixa, o que acarretaria maior tolerância e/ou potência no exercício anaeróbio devido à melhor eficácia no tamponamento celular ${ }^{(28,29)}$.

Embora a maior oferta de creatina ao organismo possa ter potencializado as respostas anaeróbias no grupo CRE, a restrição dos suplementos alimentares habituais para ambos os grupos (carboidratos e proteínas) e o elevado volume de treinamento durante a semana experimental possivelmente interferiram nos resultados. Por outro lado, CM mostrou ser um potente anticatabólico e que pode ser utilizada como recurso para a recuperação de atletas que praticam atividades prolongadas de elevada intensidade.

A partir dos achados do presente estudo, podemos concluir que a suplementação com CM (0,3g/kg por sete dias) aumentou o instante da potência pico no teste de Wingate em atletas de elite do mountain bike, sugerindo que a suplementação com CM pode melhorar o desempenho físico durante o trabalho de alta intensidade e curta duração.

\section{REFERÊNCIAS BIBLIOGRÁFICAS}

1. Mendes RR, Pires I, Oliveira A, Tirapegui J. Effects of creatine supplementation on the performance and body composition of competitive swimmers. J Nutr Biochem. 2004;15:473-8.

2. Volek JS, Rawson ES. Scientific basis and practical aspects of creatine supplementation for athletes. Nutrition. 2004;20:609-14.

3. Havenetidis $K$, Matsouka $O$, Cooke CB, Theodorou A. The use of varying creatine regimes on sprint cycling. J Sports Sci Med. 2003;2:88-97.

4. Smith SA, Montain SJ, Zientara GP, Fielding RA. Use of phosphocreatine kinetics to determine the influence of creatine on muscle mitochondrial respiration: an in vivo 31P-MRS study of oral creatine ingestion. J Appl Physiol. 2004;96:2288-92.

5. Hultman E, Sahlin K. Acid-base balance during exercise. Exerc Sport Sci Rev. 1980;8:41-128.

6. Impellizzeri FM, Marcora SM, Rampinini E, Mognoni P, Sassi A. Exercise intensity during off-road cycling. Med Sci Sports Exerc. 2002;36:1808-13

7. Havenetidis K, Matsouka O, Konstadinou V. Establishment of the highest peak anaerobic power prior to the commencement of the anaerobic Wingate test. J Hum Mov Stud. 2003;44:479-87.

8. Green AL, Hultman E, MacDonald IA, Sewell DA, Greennhaff PL. Carbohydrate ingestion augments skeletal muscle creatine accumulation during creatine supplementation in humans. Am J Physiol. 1996;271:821-6.

9. Vandenberghe K, Gillis N, Van Leemputte M, Van Hecke P, Vanstapel F, Hespel, P. Caffeine counteracts the ergogenic action of muscle creatine loading. J Appl Physiol. 1996;80:452-7.

10. Brozek J, Grande F, Anderson JT, Keys A. Densitometric analysis of body composition: revision of some quantitative assumptions. Ann N Y Acad Sci. 1963;110:113-40.

11. Weast C. Chemistriy and Physics. 69 Edição. Editora Boca Raton, Florida, 1989.

12. Goldman HI, Becklake MR. Respiratory function tests: normal values of medium altitudes and the prediction of normal results. Am Rev Respir Dis. 1959;79:457-67.

13. Buskirk ER. Body composition analysis: the past, the present and future. Exercise Sports, 1961.

14. Siri WE. Techniques for measuring body composition. National Academicy of Science 1961:233-44

15. Izquierdo M, Ibanez J, Gonzalez BJ, Gorostiaga EM. Effects of creatine supplementation on muscle power, endurance, and sprint performance. Med Sci Sports Exerc. 2002;34:332-43.

16. Waber JP, Tharion WJ, Patton JF, Champagne CM, Mitotti P, Lieberman HR. The effect of creatine supplementation multiple bench on obstacle course and press performance. J Strength Cond Res. 2002;16:500-8.
17. Deldicquev L, Louis M, Theisen D, Nielens H, Dehoux M, Thissen JP, et al. Increased IGF mRNA in human skeletal muscle after creatine supplementation. Med Sci Sports Exerc. 2005;37:731-6.

18. Olsen S, Aagaard P, Kadi F, Tufekovic G, Verney J, Olesen JL, et al. Creatine supplementation augments the increase in sattelite cell and myonuclei number in human skeletal muscle induced by strength training. J Physiol. 2006:107-359.

19. Goldbherg AL, Chang TW. Regulation and significance of amino acid in skeletal muscle. Fed Proc. 1978;37:2301-7.

20. Kettelhut IC, Wing SS, Goldbeherg AL. Endocrine regulation of protein breakdown in skeletal muscle. Diab Metab Rev. 1988:4:751-72.

21. Casey A, Grenhaff P. Does dietary creatine supplementation play a role in skeletal muscle metabolism and performance? Am J Clin Nutr. 2000;72:6075-617S.

22. Tarnopolsky MA, Maclennan DP. Creatine monohydrate supplementation enhances high-intensity exercise performance in males and females. Int J Sport Nutr Exerc Metab. 2000;10:452-63.

23. Reen D, Dawson B, Goodman C, Lawrence S, Beilby J, Ching S. Effect of creatine loading on long-term sprint exercise performance and metabolism. Med Sci Sports Exerc. 2001;33:814-21.

24. Eckerson JN, Stout JR, Moore GA, Stone NJ, Iwan KA, Gerbauer N, et al. Effect of creatine phosphate supplementation on anaerobic working capacity and body weight after two and six days of loading in men and women. J Sci Med Sport. 2005;8:305-13.

25. Kurosawa Y, Hamaoka T, Katsumura T, Kuwamori M, Kimura N, Sato T, et al. Creatine supplementation enhances anaerobic ATP synthesis during a single $10 \mathrm{sec}$ maximal handgrip exercise. Mol Cel Biochem. 2003;244:105-12.

26. Bogadanis GC, Nevill ME, Boobis LH, Lakomy HK, Neill AM. Recovery of power output and muscle metabolism following $30 \mathrm{~s}$ of maximal sprint cycling in man. J Physiol. 1998;482:467-80

27. Deutekom M, Beltman JG, Koning JJ, Haan A. No acute effects of short-term creatine supplementation on muscle properties and sprint performance. Eur J Appl Physiol. 2000;82:223-9.

28. Chetlin R, Schoenleber J, Bryner R, Gordon P, Ulrich I, Yeater R. The effects of two forms of oral creatine supplementation on anaerobic performance during the Wingate test. J Strength Cond Res. 1998;12:273

29. Hoffman JR, Stout J, Falvo MJ, Kang J, Ratmess NA. Effect of low - dose, short duration creatine supplementation on anaerobic exercise performance. J Strength Cond Res. 2005;19:260-4. 